\section{Kikuchi-Fujimoto disease associated with hemo- phagocytic lymphohistiocytosis following the BNT162b2 mRNA COVID-19 vaccination}

Kikuchi-Fujimoto disease (KD) is a self-limiting histiocytic necrotizing lymphadenitis. One case of KD following administration of the BNT162b2 mRNA COVID-19 vaccine was recently reported. ${ }^{1}$ Hemophagocytic lymphohistiocytosis (HLH) is a life-threatening hyperinflammatory state brought on by uncontrolled histiocytes, macrophages and T-cell activation, which have also been occasionally observed after BNT162b2 vaccination. ${ }^{2} \mathrm{KD}$ and HLH present overlapping pathogenesis and symptoms, and their association has been previously described in children and adult patients. ${ }^{3,4}$ Here, we report the first case of KD associated with HLH following the BNT162b2 mRNA COVID-19 vaccination.

A 38-year-old previously healthy woman was admitted to the hospital with a history of a fever of $40^{\circ} \mathrm{C}$ for more than ten days, associated with chills and fatigue. She presented with a diffuse cutaneous eruption of erythematous papules, which were subsequently confluent (Figure 1, AB). She had previously been treated with antibiotics (amoxicillin and clavulanic acid, followed by teicoplanin and doxycycline), with no improvement. The first dose of the BNT162b2 mRNA COVID-19 vaccine was administered two months after giving birth to a healthy baby. Three weeks before the onset of fever, the patient received a second dose of the vaccine, inoculated in the same left arm as the first dose. At hospital admission, her nasopharyngeal swab for SARS CoV-2 PCR was negative; SARS CoV-2 IgG antibodies were positive $(>2.080$ BAU/mL; cut-off: 33.8 BAU/mL; LIAISON SARS-CoV-2 TrimericS IgG, Diasorin, Saluggia, Italy).

Her physical exam was normal except for the cutaneous rash and multiple enlarged tender lymph nodes in the left axillary zone. This was confirmed by a contrastenhanced computed tomography (CT) examination (Figure 1, C-D).

Laboratory tests showed bi-cytopenia with leukopenia and anemia (neutrophil count, $0.9 \times 10^{9} / \mathrm{L}$; lymphocyte count, $0.3 \times 10^{9} / \mathrm{L}$, hemoglobin, $9.8 \mathrm{~g} / \mathrm{L}$ ), increased lactate dehydrogenase and transaminase levels, high serum ferritin levels $(500 \mu \mathrm{g} / \mathrm{L})$, mild hypertriglyceridemia (225 $\mathrm{mg} / \mathrm{dL}$ ) and normal fibrinogen. Her serum soluble interleukin-2 receptor (IL-2R) level was increased to 2.610 $\mathrm{U} / \mathrm{mL}$ (normal value 223-710) and her natural killer (NK) cell count was low ( $<35$ cells $/ \mu \mathrm{L}$; normal value $200-400)$. IL-6, IL- 8 and IL-10 levels were normal, but the tumor necrosis factor (TNF) level was increased. The patient underwent a bone marrow aspiration and trephine proce-

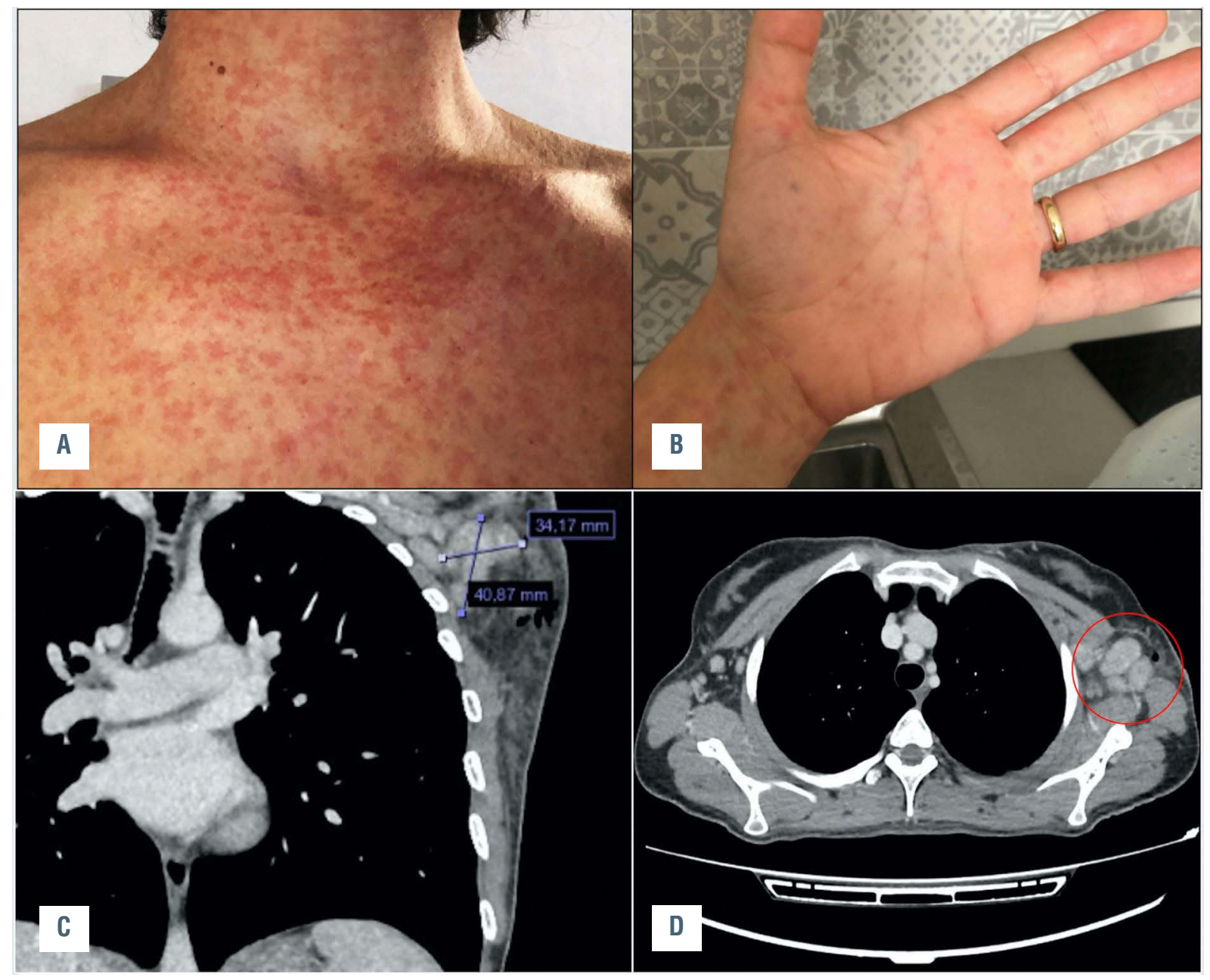

Figure 1. Patient's clinical and radiological findings. A and B) Diffuse maculo-papular skin rash; C and D) CT scan showing left axillary lymph node enlargement. 
dure, which showed hemophagocytosis (Figure 2, A-C) A diagnosis of HLH was confirmed based on the fulfilment of 6/8 HLH-2004 diagnostic criteria. ${ }^{5}$ In addition, an excisional lymph node biopsy of the left axillary was performed. Histopathological examination of the lymph node revealed histiocytic necrotizing lymphadenitis, characterized by paracortical, well-circumscribed necrotic areas with karyorrhexis and fibrin deposits. Immunohistochemistry revealed numerous CD68-positive histiocytes as well as several CD3-positive $\mathrm{T}$ cells and few CD20-positive B cells. All these features are considered typical of KD (Figure 2, D-F).

Further analysis did not show ongoing infections of HCV, HBV hepatitis virus, HIV, toxoplasma, rubeovirus, brucella, leptospirosis, bartonella, chlamydia, morbillivirus, mycoplasma or yersinia. The tuberculosis Quantiferon test was also negative. Epstein Barr virus (EBV), Parvovirus B19, cytomegalovirus, JC virus and herpes-6 DNA were also absent. Antinuclear antibodies (ANA), antibodies to double-stranded DNA (antidsDNA), and antibodies to extractable nuclear antigens (anti-ENA) were absent. The rheumatoid factor test was negative and both complement C3 and C4 serum levels were normal.

The patient was promptly initiated on steroids (methylprednisolone $1 \mathrm{mg} / \mathrm{kg}$ i.v.), in adherence with the recommended treatment for HLH. Sudden fever lysis was observed and clinical conditions improved. Methyl-pred-

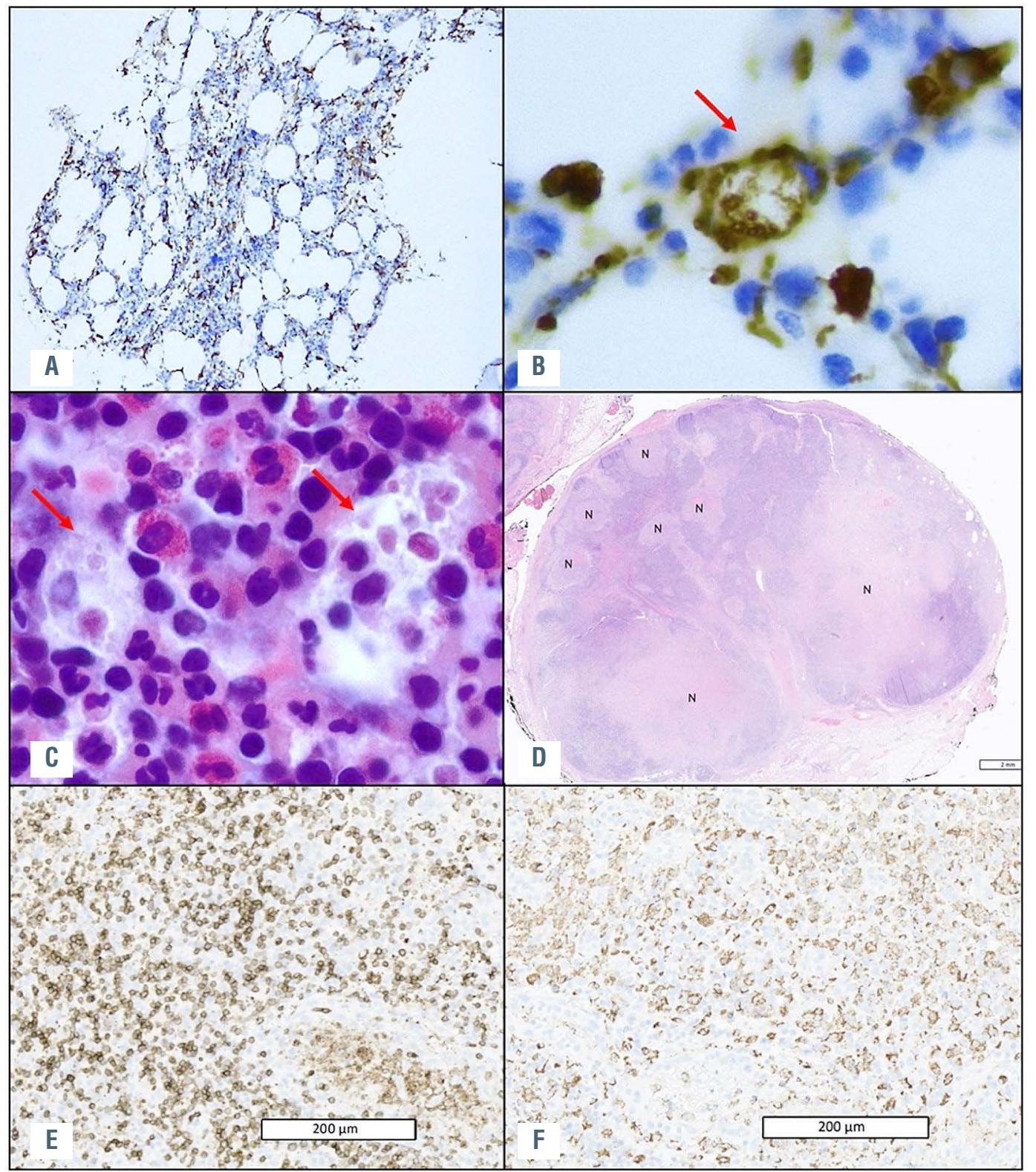

Figure 2. Lymph node and bone marrow histological findings. A) Increased diffusion of histiocytes (brown color) in the bone marrow trephine biopsy; CD68 (PGM1) stain, 10X magnification; B) An histiocyte showing phagocytosis of hemopoietic elements; CD68 (PGM1) stain, 60X magnification; C) Histiocytes showing phagocytosis of red cells, platelets, erythroid precursor cells; hematoxylin-eosin stain, 60X magnification; D) A low power view of the lymph node showing paracortical necrotizing zones $(\mathrm{N})$ in an otherwise preserved architecture; hematoxylin-eosin stain; E) The paracortical necrotizing areas were characterized by many reactive T lymphocytes, as shown by immunohistochemistry for CD3; 10X magnification; F) The immunostaining for CD68 revealed numerous histiocytes within the necrosis; 10X magnification. 
nisone was substituted with prednisone $(1 \mathrm{mg} /$ day) at hospital discharge one week later. This schedule was maintained for a further two weeks, reduced to a half dosage for three more weeks, then tapered over the following three weeks. IL-2R levels decreased to 1.170 $\mathrm{U} / \mathrm{mL}$ and $740 \mathrm{U} / \mathrm{mL}$ after two and three weeks of steroid treatment, respectively, and reached normal levels (460 $\mathrm{U} / \mathrm{mL}$ ) after four weeks. NK cells also returned to normal levels. The patient is doing well and follow-up is still ongoing.

To the best of our knowledge, this is the first case of $\mathrm{KD}$ associated with HLH following COVID-19 vaccination. HLH is a severe hyperinflammatory syndrome, secondary to aberrant cytokine production and uncontrolled histiocyte activation, following infections, in particular EBV, hematological malignancies, autoimmune diseases, hematopoietic stem cells or organ transplantation. It is characterized by cytopenia, unremitting fever, hepatic dysfunction and a fatal multiple organ failure without early recognition and prompt treatment. The first line of treatment is steroids and/or immunoglobulins, with etoposide added in poor responders. ${ }^{5} \mathrm{KD}$ is a self-limiting histiocytic necrotizing lymphadenitis that commonly occurs in Asia, although it is present globally. ${ }^{6}$ It is characterized by fever, lymphadenopathy and leukopenia, may be associated with a skin rash and is mainly characterized by transient red, millet-sized maculopapules.? Since no skin biopsy was carried out, it remains unclear whether the patient's skin rash was associated with $\mathrm{KD}$ or was a possible adverse effect of teicoplanin (Figure 1, A-B). KD pathogenesis is unknown, although it is believed to be a consequence of an aberrant $T$ cells and histiocyte immune response to an immunogenic antigen. An association with organisms such as toxoplasma, cytomegalovirus, varicella-zoster virus, EBV, human herpes virus-6, HIV, and yersinia enterocolitica, has been suggested, although convincing evidence has yet to be presented. Moreover, a possible association with autoimmune disorders, including antiphospholipid antibody syndrome and mixed connective tissue disease, has been reported. ${ }^{1}$ Steroids and immunoglobulins treatment may be beneficial. The association between HLH and KD has been described in both children and adult patients and has a potentially fatal outcome if left untreated..$^{3,4}$ Some authors have reported that that CD8+ T lymphocytes in patients with HLH-KD may be excessively activated, altering the course of the self-limited $\mathrm{KD}$ progress and resulting in $\mathrm{HLH}^{8}$

Both HLH and KD following BNT162b2 mRNA COVID-19 vaccination have recently been described. ${ }^{1,2}$ Notably, after mass vaccination against COVID-19, a vaccine-associated hypermetabolic lymphadenopathy (VAHL) in the axillary or supraclavicular lymph nodes, ipsilateral to the vaccination site, has been reported..$^{9,10,11}$ This is frequently observed after BNT162b2 administration, with higher intensities following the booster dose, and lasting until three weeks after vaccination. We hypothesize that our patient first developed a left-axillary VHAL following two ipsilateral vaccine dose inoculations, followed by a systemic inflammatory response syndrome (SIRS) with KD features in the axillary lymph nodes, and HLH symptoms as a systemic inflammatory reaction. Dermal histiocytes and macrophages represent the first resident antigen-presenting cell (APC) transfected by the mRNA vaccine that presents antigenic peptides on major histocompatibility (MHC) class I and MHC class II molecules of CD4+ and CD8+ cells, resulting in immune response expansion. Additionally, intramuscular vaccine injection leads to a local increase in proinflamma- tory cytokines, which form an immune-stimulatory environment in draining axillary lymph nodes. ${ }^{12}$ It remains unclear whether an alternate arm inoculation in our patient might have been less immune-reactive.

In conclusion, we have described the first case of $\mathrm{KD}$ associated with HLH following COVID-19 vaccination. This is a rare event and does not compromise the safety and efficacy of the BNT162b2 mRNA vaccine in the fight against COVID-19. Physicians should be aware of rare systemic inflammatory reactions that require early diagnosis and treatment.

Giovanni Caocci, ${ }^{1}$ Daniela Fanni, ${ }^{2}$ Mariagrazia Porru, ${ }^{3}$ Marianna Greco, 1 Sonia Nemolato, ${ }^{2}$ Davide Firinu, ${ }^{3}$ Gavino Faa, ${ }^{2}$ Angelo Scuteri ${ }^{3}$ and Giorgio La Nasa ${ }^{1}$

${ }^{1}$ Department of Medical Sciences and Public Health, Haematology, University of Cagliari, Businco Hospital; 2 Department of Medical Sciences and Public Health, Pathology, University of Cagliari,

S. Giovanni di Dio and Businco Hospital and ${ }^{3}$ Department of Medical Sciences and Public Health, Internal Medicine, University of Cagliari,

Policlinico Hospital, Cagliari, Italy

Correspondence:

GIOVANNI CAOCCI - giovanni.caocci@unica.it

doi:10.3324/haematol.2021.280239

Received: October 25, 2021.

Accepted: December 20, 2021

Pre-published: December 30, 2021.

Disclosures: no conflicts of interest to disclose.

Contributions: GC, GF, AS and GLN conceived and designed the study; GC, MP, DF, AS and GLN managed patients; DF, SN and GF carried out the pathological analysis; MG carried out the immunophenotype and interleukin analysis: GC wrote the manuscript; $G C, D F, M P, D F, M G, S N, G F, A S, G L N$ approved the final draft of the manuscript.

Acknowledgments: we thank Dr. Mariano Cabiddu and Dr. Giovanna Manconi for patient management in the early phases; Prof. Fabio Medas for performing lymph node biopsy; Dr. Salvatore Labate for CT images and Dr. Valeria Fresu for interleukin analysis.

Compliance with ethical standards: all procedures performed in studies involving human participants were in accordance with the ethical standards of the institutional and/or national research committee and with the 1964 Helsinki declaration and its later amendments or comparable ethical standards

Data sharing statement: the data that support the findings of this study are available from the corresponding author, upon reasonable request.

Informed consent: informed consent was obtained from the patient, including the publication of personal images..

\section{References}

1. Soub HA, Ibrahim W, Maslamani MA, A Ali G, Ummer W, AbuDayeh A. Kikuchi-Fujimoto disease following SARS CoV2 vaccination: Case report. IDCases. 2021;25:e01253.

2. Tang LV, Hu Y. Hemophagocytic lymphohistiocytosis after COVID19 vaccination. J Hematol Oncol. 2021;14(1):87.

3. Nishiwaki M, Hagiya H, Kamiya T. Kikuchi-Fujimoto disease complicated with reactive hemophagocytic lymphohistiocytosis. Acta Med Okayama. 2016;70(5):383-388.

4. Duan W, Xiao Z-H, Yang L-G, Luo H-Y. Kikuchi's disease with hemophagocytic lymphohistiocytosis: a case report and literature review. Medicine (Baltimore). 2020;99(51):e23500.

5. Henter J-I, Horne A, Aricó M, et al. HLH-2004: Diagnostic and therapeutic guidelines for hemophagocytic lymphohistiocytosis. Pediatr Blood Cancer. 2007;48(2):124-131.

6. Perry AM, Choi SM. Kikuchi-Fujimoto Disease: A Review. Arch Pathol Lab Med. 2018;142(11):1341-1346. 
7. Resende C, Araújo C, Duarte M da L, Vieira AP, Brito C. Kikuchi's disease of the xanthomathous type with cutaneous manifestations. An Bras Dermatol. 2015;90(2):245-247.

8. Yang Y, Lian H, Ma H, et al. Hemophagocytic lymphohistiocytosis associated with histiocytic necrotizing lymphadenitis: A clinical study of 13 children and literature review. J Pediatr. 2021;229:267274.

9. Cohen D, Krauthammer SH, Wolf I, Even-Sapir E. Hypermetabolic lymphadenopathy following administration of BNT162b2 mRNA Covid-19 vaccine: incidence assessed by [18F]FDG PET-CT and relevance to study interpretation. Eur J Nucl Med Mol Imaging.
2021;48(6):1854-1863.

10. Steinberg J, Thomas A, Iravani A. 18F-fluorodeoxyglucose PET/CT findings in a systemic inflammatory response syndrome after COVID-19 vaccine. Lancet. 2021;397(10279):e9.

11. Adin ME, Isufi E, Kulon M, Pucar D. Association of COVID-19 mRNA vaccine with ipsilateral axillary lymph node reactivity on imaging. JAMA Oncol. 2021;7(8):1241-1242

12. Mascellino MT, Di Timoteo F, De Angelis M, Oliva A. Overview of the main anti-SARS-CoV-2 vaccines: mechanism of action, efficacy and safety. Infect Drug Resist. 2021;14:3459-3476. 